\title{
Pleckstrin homology-like domain, family A, member 1 (PHLDAI) and cancer (Review)
}

\author{
MARIA APARECIDA NAGAI \\ Discipline of Oncology, Department of Radiology, Faculty of Medicine, University of São Paulo, \\ Laboratory of Molecular Genetics, Center for Translational Research in Oncology, \\ Cancer Institute of São Paulo, São Paulo, SP 01246-000, Brazil
}

Received January 11, 2016; Accepted January 19, 2016

DOI: $10.3892 /$ br. 2016.580

\begin{abstract}
Pleckstrin homology-like domain, family A, member 1 (PHLDA1) encodes a member of an evolutionarily conserved pleckstrin homology-related domain protein family. It was first identified as a potential transcription factor required for Fas expression and activation-induced apoptosis in mouse $\mathrm{T}$ cell hybridomas. The exact molecular and biological functions of PHLDAl remain to be elucidated. However, its expression is induced by a variety of external stimuli and there is evidence that it may function as a transcriptional activator that acts as a mediator of apoptosis, proliferation, differentiation and cell migration dependent on the cellular type and context. Recently, PHLDA1 has received attention due to its association with cancer. In the present review, the current knowledge of PHLDA1 protein structure, expression regulation and function is summarized. In addition, the current data in the literature is reviewed with regards to the role of PHLDA1 in cancer pathogenesis.
\end{abstract}

Correspondence to: Dr Maria Aparecida Nagai, Discipline of Oncology, Department of Radiology, Faculty of Medicine, University of São Paulo, Laboratory of Molecular Genetics, Center for Translational Research in Oncology, Cancer Institute of São Paulo, Avenida Dr Arnaldo 251, 8th Floor, São Paulo, SP 01246-000, Brazil

E-mail: nagai@usp.br

Abbreviations: aa, amino acid; AICD, activation-induced cell death; BCC, basal cell carcinoma; EMT, epithelial-mesenchymal transition; ER, endoplasmic reticulum; FGF, fibroblast growth factor; IGF-1, insulin-like growth factor 1 ; NF- $\kappa \mathrm{B}$, nuclear factor- $\kappa \mathrm{B}$; OSCC, oral squamous cell carcinoma; PH, proline-histidine; PHL, pleckstrin homology-like; PHLDA1, pleckstrin homology-like domain, family $\mathrm{A}$, member 1 ; $\mathrm{PKC}$, protein kinase $\mathrm{C}$; $\mathrm{PQ}$, proline-glutamine; QQ, polyglutamine

Key words: PHLDA1, pleckstrin homology-like, cancer, tumor suppressor gene, oncogene, downregulation, stem cell

\section{Contents}

1. Introduction

2. PHLDA1 expression and cell death

3. Role of PHLDA1 in cancer

4. Conclusions

\section{Introduction}

Pleckstrin homology-like domain, family A, member 1 (PHLDA1; also known as PHRIP, TDAG51, DT1P1B11 and MGC131738) was first identified as a potential transcription factor required for Fas expression and activation-induced apoptosis in mouse $\mathrm{T}$ cell hybridomas (1). It was later mapped to human chromosome $12 \mathrm{q} 15$ by radiation hybrid mapping (2). The PHLDA family is evolutionarily conserved and comprises three members that are located on different chromosomes: PHLDA1 (12q21.2), PHLDA2 (11p15.4) and PHLDA3 (1q32.1). All three share similar gene organization [i.e., two exons (one coding exon) and a small intron] and encode proteins with a PHL domain (3). During the last decade, PHLDA1 has received increasing attention, although its functional role remains to be elucidated.

PHLDAl encodes a protein that is 401 amino acids (aa) in length. This protein is rich in polyglutamine (QQ), proline-glutamine (PQ) and proline-histidine tracts with a PHL domain (1-3) (Fig. 1). Sequencing comparisons have shown that mouse, human and rat PHLDA1 cDNA and protein exhibit high levels of sequence identity $(2,4)$.

PHL domains are evolutionarily conserved and are found in proteins from prokaryotes and eukaryotes $(5,6)$. These domains are composed of 100-120 aa residues, and their properties include binding to phosphatidylinositol lipids (5). Numerous proteins containing PHL domains (such as insulin receptor substrate proteins) have been found to interact with membrane components, eliciting a variety of cellular responses and participating in cell signaling transduction, vesicular trafficking and cytoskeletal rearrangement $(7,8)$. QQ and PQ stretches are well-conserved motifs in eukaryotic proteins, and they are present predominantly in transcription factors that are involved in transcriptional activation and protein-protein interactions $(9,10)$. In addition, a number of variations in 
tandem repeats of the trinucleotide sequence $\mathrm{CAG}$, which encodes homopolymeric stretches of QQ, have been associated with several inherited neurodegenerative diseases $(10,11)$. Due to the presence of QQ stretches that function as transcriptional activation domains, PHLDA1 could act as a transcription factor required for Fas expression (1).

\section{PHLDA1 expression and cell death}

PHLDA1 mRNA and protein are expressed in several mammalian tissues, suggesting that it may have important cellular functions $(1,4,12)$. In addition to being widely expressed in different tissues, PHLDA1 subcellular localization varies. PHLDA1 is found predominantly in the cytoplasm (13-16); however, perinuclear, nucleolar and nuclear localization have also been reported $(12,13,16,17)$. PHLDA1 expression is induced by different stimuli, such as estrogens, growth factors, differentiation and endoplasmic reticulum (ER)-stress agents, and it is associated with different biological processes, such as cell apoptosis, cell proliferation and differentiation (Fig. 2).

Evasion of apoptosis or programmed cell death is a hallmark of cancer, and the development of resistance to apoptosis is associated with tumor pathogenesis and affects chemo- and radioresistance (18). Several lines of experimental evidence have implicated PHLDA1 in intrinsic and extrinsic apoptotic pathways; however, the mechanisms involved in its action as a proapoptotic or antiapoptotic agent remain to be elucidated.

The role of PHLDA1 in activation-induced cell death (AICD) was first reported by Park et al (1) in 1996, who used a T cell hybridoma model to determine that PHLDA1 expression is induced by $\mathrm{T}$ cell receptor activation in the presence of phorbol myristate acetate and ionomycin (1). The study also reported that PHLDA1 is required for Fas (CD95) expression, which has an important role in $\mathrm{T}$ cell receptor-induced apoptosis. These data point to an essential role for PHLDA1 in the induction of apoptosis in a T cell hybridoma model. However, the study by Rho et al (19) investigated the assocation between PHLDA1 and Fas expression in vivo and demonstrated that PHLDA $1^{-1-}$ mice express normal levels of Fas and have normal $\mathrm{T}$ cell apoptosis, indicating that PHLDA1 is not essential for Fas expression. Furthermore, Oberg et al (20) demonstrated that in human $\mathrm{T}$ cells and pancreatic tumor cells, induction of PHLDA1 is inhibited by mitogen-activated protein kinase (MAPK) and protein kinase C (PKC) inhibitors, indicating that these two pathways may be involved in the regulation of PHLDA1 expression. However, no association was found between PHLDA1 expression and AICD or cell proliferation. By contrast, increased Fas expression was shown to be dependent on PKC activation $(1,21)$, which induces Fas expression only in cells expressing wild-type PHLDA1 (21). Sequence analysis and experimental studies revealed that PHLDA1 contains putative casein kinase II and PKC phosphorylation sites $(4,21)$. These data suggest that PHLDA1 transcriptional activation of the target gene FAS may be dependent on post-transcriptional modification upon PKC activation, which raises the possibility of transcriptional activity of PHLDA1 in a broader context.

In agreement with the proapoptotic role executed by PHLDA1 in rat neuronal cells, PHLDA1 mRNA and protein were induced by fibroblast growth factor and phorbol dibutyrate, which is a differentiation agent that activates PKC (4). In addition, Gomes et al (4) reported that microinjection of differentiating H19-7 hippocampal cells with the neutralizing antibody anti-PHLDA1 increases cell survival. Furthermore, transient PHLDA1 expression decreases cell survival in differentiating and non-differentiating H19-7 cells, indicating that PHLDA1 acts as a mediator of differentiation-associated apoptosis in H19-7 cells (4). PHLDA1 expression is induced by the phorbol ester and ionomycin in different transformed $\mathrm{T}$ cell lines and was also found to interact with two mRNA-binding proteins, eIF3-p66 and PABP, in 293T cells. Additionally, PHLDA1 cotransfection with the luciferase gene leads to protein biosynthesis inhibition, a process that is important for the execution of apoptosis (13).

ER stress-inducing agents, such as homocysteine, tunicamicyn and farnesol (FOH), have been reported to induce PHLDA1 expression in different cell types $(12,22,23)$. Hossain et al (12) demonstrated that induction of PHLDA1 by homocysteine involves ER stress and is dependent on eIF2 $\alpha$ phosphorylation. In addition, transient overexpression of PHLDA1 leads to changes in cell morphology, decreases cell adhesion, and promotes detachment-mediated cell death in endothelial cells (12). To improve the understand how FOH induces apoptosis, Joo et al (22) performed cDNA microarray analysis and compared the gene expression profiles between vehicle-treated and FOH-treated H460 human lung adenocarcinoma cells. An induction of a number of genes associated with ER stress-response signaling, including PHLDA1, was observed. The study also reported that FOH-induced apoptosis in human lung carcinoma cells is coupled to the activation of an ER-stress response that includes activation of the sensors IRE1 and PERK, dependent on the activation of the MEK-ERK signaling pathway.

Carlisle et al (23) found that treatment of HK-2 cells (an immortalized proximal tubule epithelial cell line) with the ER-stress agents thapsigargin and cyclosporine A increases the cytosolic $\mathrm{Ca}^{2+}$ concentration and induces PHLDA1 expression, leading to epithelial-mesenchymal transition (EMT). In addition, overexpression of PHLDA1 in HK-2 cells leads to shape changes, $\beta$-catenin disruption and increased apoptosis, indicating that PHLDA1 may cause dissolution of adherens junctions, $\beta$-catenin nuclear localization and EMT induction (23). EMT is an important biological process that involves downregulation of epithelial markers and upregulation of mesenchymal markers, in which epithelial cells lose cell-cell adhesion and cell polarity, and gain the migratory and invasive properties that are characteristic of mesenchymal cells $(24,25)$. As EMT has a key role in tumor progression and may influence therapy response, the potential role of PHLDA1 as an EMT regulator requires further investigation.

In support of the proapoptotic function exhibited by PHLDA1, Neef et al (14) reported that constitutive PHLDA1 expression in different melanoma-derived cell lines leads to reduced cell growth, reduced colony formation and increased basal apoptosis, which is associated with increased cleaved caspase 9 and cleaved poly adenosine diphosphate ribose polymerase. Upregulation of PHLDA1 has been identified in autophagy and apoptosis induced by rapamycin in T47D breast cancer cells (26). In addition, stress-induced heat-shock proteins (Hsp; including Hsp70, Hsp110 and Hsp40) bind 


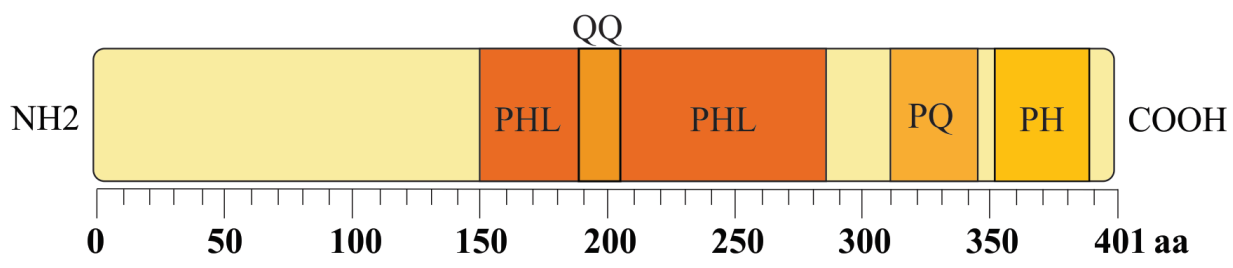

Figure 1. Schematic representation of the modular structure of the PHLDA1 protein. PHL, pleckstrin homology-like domain (aa 151-283); QQ, polyglutamine tract (aa 187-204); PQ, proline-glutamine tract (aa 311-346); PH, proline-histidine-rich tract (aa 352-389); aa, amino acid.

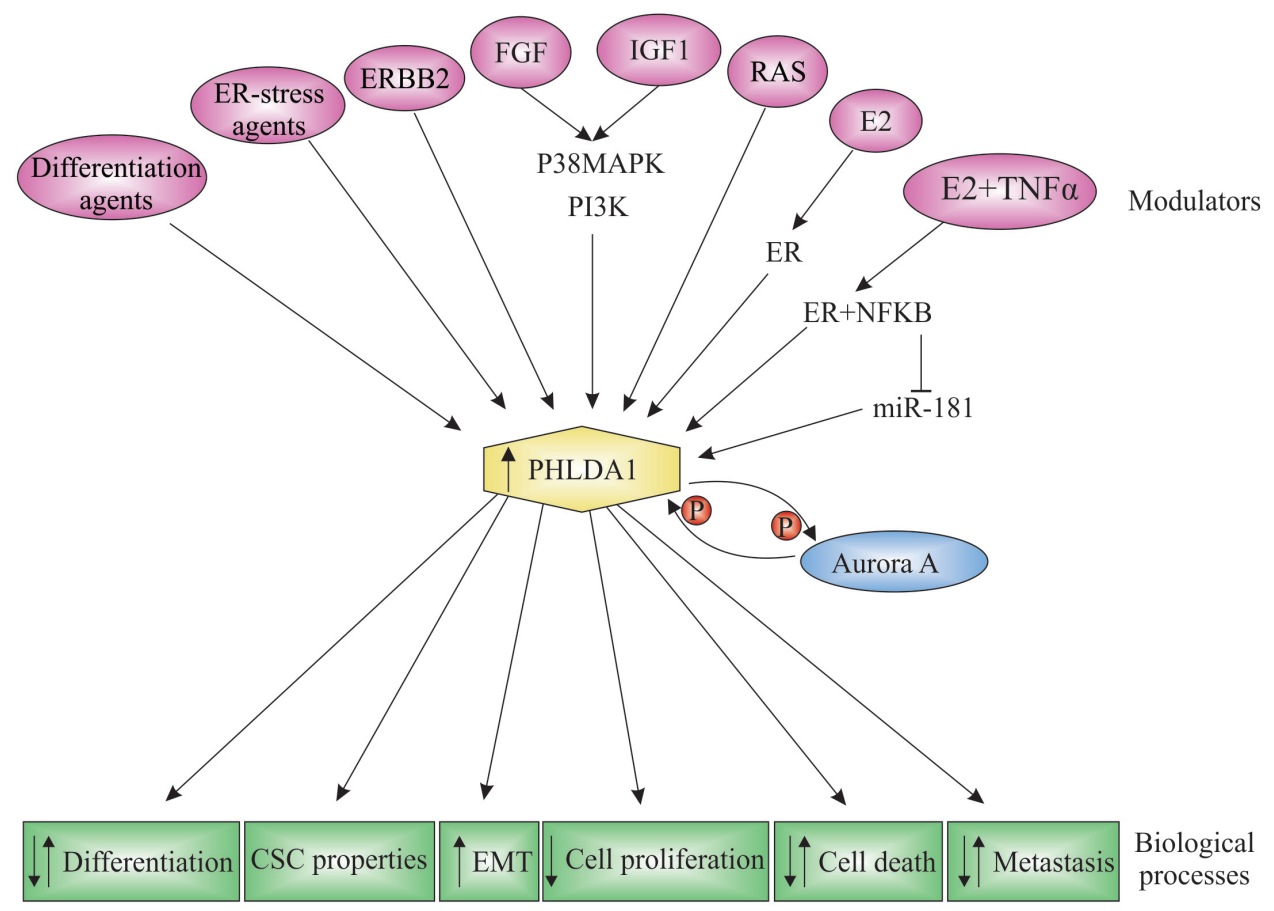

Figure 2. Modulators and biological functions of Pleckstrin homology-like domain, family A, member 1 (PHLDA1). PHLDA1 expression is modulated by a variety of external stimuli in different cell types. As a consequence, diverse biological processes are modulated. ER-stress agents, endoplasmic reticulum-stress agents; ERBB2, receptor tyrosine kinase 2; P, phosphorylation; FGF, fibroblast growth factor; IGF1, insulin-like growth factor 1; RAS, rat sarcoma virus oncogene; E2, 17 $\beta$-estradiol; ER, estrogen receptor; NF- $\mathrm{KB}$, nuclear factor of $\kappa$ light polypeptide gene enhancer in B cells; p38 MAPK, p38 mitogen-activated protein kinase; PI3K, phosphatidylinositol 3-kinase; TNF- $\alpha$, tumor necrosis factor- $\alpha$; miR-181, microRNA 181.

directly to the pleckstrin domain of PHLDA1 and suppress its proapoptotic function in mouse embryonic fibroblast (MEF) cells (27). The promoter region of PHLDAl has heat-shock element binding sites, where heat-shock transcription factor 1 (HSF1) binds and modulates PHLDAl expression. In addition, HSF1 is able to directly bind to and activate PHLDAl in response to heat shock in male germ cells (27). Furthermore, during a time-course induction of cryptorchidism in rat testes, HSF1 and PHLDA1 were strongly expressed, suggesting that the HSF1/PHLDA1 pathways have an important role in the apoptosis of primary spermatocytes in cryptorchid testes (28).

In addition to its association with apoptosis induction, PHLDA1 has also been shown to act as an antiapoptotic agent. PHLDA1 mRNA and protein are induced by insulin-like growth factor 1 (IGF-1) through IGF-1R via the p38 MAPK pathway in NIH-3T3 (NWTb3) cells and via the phosphatidylinositol 3-kinase pathway in normal skin fibroblasts $(29,30)$. Knockdown of PHLDAl expression by small interfering RNA (siRNA) abolishes the protective effects of IGF-1 against apoptosis induction in NIH-3T3 (NWTb3) cells in response to serum starvation (30). Toyoshima et al (29) also identified that PHLDAl downregulation increases apoptosis, which is independent of IGF-1, suggesting that PHLDA1 may have antiapoptotic effects in NWTb3 cells.

PHLDA1 is also a direct target of the oncogenic fusion protein EWS/FLI1. Boro et al (31) demonstrated that EWS/FLI1 can bind to the promoter of PHLDA1, in vivo and in vitro, leading to its repression. In MEF cells derived from conditional EWS/FLI1 knockin embryos, EWS/FLI1 expression resulted in apoptosis and EWS/FLI1 was able to directly bind the caspase 3 promoter in CHP100 cells with a concomitant increase in endogenous caspase 3 expression (32). In addition, PHLDAl knockdown by siRNA in Ca9-22 cells increases activated caspase 3 expression, suggesting that PHLDA1 has a role as an apoptosis suppressor (33).

\section{Role of PHLDA1 in cancer}

Several lines of evidence suggest that PHLDA1 has an important role in cancer. Although the exact molecular and cellular functions of PHLDA1 have not been fully elucidated, evidence from experimental studies show that it is a potential 
Table I. Aberrant expression of PHLDA1 in cancer.

\begin{tabular}{ll}
\hline Type of cancer & \multicolumn{1}{c}{ Expression } \\
\hline Melanoma & $\begin{array}{l}\text { Strongly expressed in benign melanocytic nevi } \\
\text { and progressively reduced in primary and } \\
\text { metastatic melanomas }\end{array}$ \\
& $\begin{array}{l}\text { Reduced expression, a strong predictor of poor } \\
\text { prognosis for breast cancer patients } \\
\text { Reduced expression associated with estrogen } \\
\text { receptor expression in breast tumors } \\
\text { Elevated expression associated with a higher } \\
\text { risk of distant metastasis in estrogen } \\
\text { receptor-positive breast cancer patients }\end{array}$
\end{tabular}

PHLDA1 regulated by E2 and TNF- $\alpha$ at the transcriptional and post-transcriptional level in breast

Expression reduced in ERBB2-negative tumor samples and ERBB2-negative breast cancer cell lines

Oral

Reduced expression is associated with good prognosis in oral cancer

Gastric

Reduced expression associated with shorter overall survival in gastric cancer patients

Basal cell Reduced expression in basal cell carcinoma carcinoma

Colorectal

Overexpressed in colorectal cancer

Intestinal

Overexpressed at all stages of intestinal cancer development

Osteosarcoma cancer cells

Silencing affects mammosphere formation and growth Reduced expression during ras-mediated cellular transformation induces proliferation under anchorage-independent conditions and increases ERK activation

Upregulation found in authophagy and apoptosis induced by rapamycin in breast cancer cells

Expression in melanoma cell lines is associated with reduced cell growth and colony formation, and increased basal apoptosis

Regulatory feedback loop between

Aurora-A and PHLDA1

Overexpression inhibits cell proliferation, colony-forming ability, and migration in SKBR3 cells

Silencing leads to apoptosis induction

Silencing in colon cancer cells inhibits anchorage-independent cell growth and reduces cell migration

High expression associated high metastatic potential in osteosarcoma cells, and silencing delays overall survival in mouse xenograft models

PHLDA1, pleckstrin homology-like domain, family A, member 1; TNF- $\alpha$, tumor necrosis factor- $\alpha$; ERK, extracellular-signal-regulated kinase; ERBB2, Erb-B2 receptor tyrosine kinase 2.

transcriptional activator that acts as a modulator of apoptosis and cell proliferation, two biological processes that impact cell survival and are considered hallmarks of cancer (34). In addition, altered expression of PHLDA1 has been described in different types of tumor, such as melanoma, breast and intestinal and gastric tumors (Table I).

Reduced PHLDA1 expression has been described in melanoma (14), breast carcinoma $(15,35,36)$, oral carcinoma (37) and gastric adenocarcinoma (16). This indicates that PHLDA1 may act as a tumor suppressor gene. However, PHLDA1 was reported to be upregulated in colon cancer and in human intestinal adenoma and carcinoma, and PHLDAl knockdown in colon cancer cells inhibits anchorage-independent cell growth and reduces cell migration $(17,38)$. Furthermore, high expression of PHLDA1 is associated with high metastatic potential in osteosarcoma cells and reduced PHLDA1 expression delays overall survival metastasis progression in mouse xenograft models (39). These findings suggest that PHLDA1 has tissue-specific functions and may have an oncogenic role in other cancer types.

In melanoma, downregulation of PHLDA1 expression was found in metastatic lesions but not in primary tumors, 
suggesting that loss of PHLDA1 expression may contribute to the progression of malignant melanoma (14). The effects of PHLDA1 expression on the biological characteristics of different melanoma-derived cell lines were also evaluated. PHLDA1 expression leads to reduced cell growth and colony formation, increased basal apoptosis, and increased sensitivity to apoptosis induced by chemotherapeutic agents (14).

Reduced expression of PHLDA1 was observed in $60.7 \%$ of oral squamous cell carcinomas (OSCC), particularly in well-differentiated tumors. Positive PHLDA1 immunostaining was associated with advanced clinical stages of the disease, suggesting that PHLDA1 has a functional role in oral tumorigenesis. Overall and disease-free survival rates were significantly improved in patients with tumors that were negative for PHLDA1, and a multivariate analysis suggested that PHLDA1 is an independent prognostic factor in OSCC patients (37).

Downregulation of PHLDA1 mRNA and protein expression is frequently observed in primary invasive breast tumors. In a large series of primary breast tumors, Nagai et al (15) reported that downregulation of the PHLDA1 protein is a strong predictor of poor prognosis for breast cancer patients. This suggests that reduced PHLDA1 expression contributes to breast cancer progression and may serve as a useful prognostic biomarker of disease outcome. The study also found that the subgroup of breast tumors that were negative for estrogen receptor and expressed PHLDA1 had a more favorable outcome. PHLDA1 downregulation has also been associated with estrogen receptor expression in breast tumors (35). In addition, high PHLDAl mRNA expression is associated with a high risk of distant metastasis in estrogen receptor-positive breast tumors. This suggests that in breast cancer cells, PHLDA1 may have different functions depending on the estrogen receptor status, which affects the clinical behavior of tumors.

In an investigation of the effects of $\mathrm{E} 2$ and the antiestrogen agent ICI 182780 on transcriptional regulation of PHLDA 1 in MCF7 breast cancer cells, Marchiori et al (40) demonstrated that PHLDA1 is upregulated by E2 via estrogen receptor in a time-dependent manner. More recently, PHLDA1 was shown to be regulated by crosstalk between ER and inflammatory nuclear factor $-\kappa \mathrm{B}(\mathrm{NF}-\kappa \mathrm{B})(41)$. PHLDA1 is regulated by $\mathrm{E} 2$ and tumor necrosis factor- $\alpha$ in MCF7 breast cancer cells through estrogen receptor and $\mathrm{NF}-\kappa \mathrm{B}$ activation. Kastrati et al (41) also found that estrogen receptor and the $\mathrm{NF}-\kappa \mathrm{B}$ pathway activation upregulates PHLDA1 directly by transcriptional activation and by indirectly repressing the expression of miR-181a and $b$, which directly target PHLDA1.

Reduction of PHLDA1 expression in HME16C mammary epithelial cells during ras-mediated cellular transformation induces proliferation under anchorage-independent conditions and results in increased ERK activation, which suggests that PHLDA1 opposes ERK-mediated transformation in breast cancer cells (42). In a profiling study with SKBR3 breast cancer cells treated with lapatinib, Li et al (36) identified PHLDA1 as a novel downstream target in the Erb-B2 receptor tyrosine kinase 2 (ERBB2)/epidermal growth factor receptor pathway and demonstrated that PHLDA1 expression is reduced in ERBB2-negative breast tumors and breast cancer cell lines. In addition, overexpression of PHLDA1 was found to inhibit proliferation, colony-formation ability and migration in SKBR3 breast cancer cells (36).

A regulatory loop between Aurora-A and PHLDA1 was also reported in breast cancer cells (35). In MDA-MB-231 breast cancer cells, Aurora-A predominantly phosphorylates PHLDA1, leading to its degradation. PHLDA1 overexpression results in decreased levels of Aurora-A, suggesting that PHLDA1 acts as a negative regulator of Aurora-A-mediated breast oncogenesis (35).

In an expression profiling study, Ohyama et al (43) determined that PHLDA1 is highly expressed and precisely localized in the bulge, which is the multipotent compartment of the hair follicle. This indicates that PHLDA1 may be an epithelial stem cell marker. The identification of PHLDA1 as a potential epithelial stem cell marker has attracted the attention of several investigators who are researching the possible role of PHLDA1 in the tumorigenic process; however, this area of investigation is in the early stages.

In an investigation of the expression patterns of PHLDA1 in 16 desmoplastic trichoepitheliomas and 14 morphoeic basal cell carcinomas (BCCs), the study by Sellheyer and Krahl (44) reported PHLDA1-positive immunostaining in $>80 \%$ of the trichoepitheliomas and PHLDA1-negative immunostaining in all the BCCs examined, indicating that expression of the hair follicle bulge marker PHLDA1 can be used to differentiate between trichoepitheliomas and BCCs. Thus, PHLDA1 could be used as a biomarker in the differential diagnosis of basaloid tumors.

Aberrant PHLDA1 expression has been described in intestinal and colorectal cancers $(17,38)$. Upregulation of PHLDA1 mRNA expression was associated with the presence of anemia in patients with colon cancer (38), and increased nuclear expression of PHLDA1 was observed in the invasive margins of human colorectal carcinomas (17). siRNA knockdown of PHLDAl expression in HCT116 and SW480 colon cancer cells under anchorage-dependent growth leads to inhibition of cell growth and colony-formation ability, and PHLDA1 downregulation also inhibits cell migration in these colon cancer cells (17). In addition, strong PHLDA1-positive immunostaining has been found in undifferentiated crypt base cells that exhibit a similar pattern of LGR5 expression. LGR5 is a putative epithelial stem cell marker for human small and large intestine (17). In agreement with this finding, Guezguez et al (45) determined that PHLDA1 is part of a primordial stem-like cell signature in normal human intestinal epithelial crypt cells treated with noggin, an antagonist to bone morphogenetic protein signaling.

In an evaluation of a large number of gastric carcinomas and normal gastric mucosa by immunohistochemisty, Zhao et al (16) observed that PHLDA1 is expressed diffusely in the cytoplasm and nucleus in crypt base cells. PHLDA1 expression appeared to mark putative epithelial stem cells in all the normal gastric mucosa analyzed. The study by Zhao et al (16) identified negative PHLDA1 expression in $57 \%$ of the gastric carcinomas and the most poorly differentiated cases were negative for PHLDA1. Strong nuclear staining was found in invasive margins. Furthermore, reduced PHLDA1 expression was associated with shorter overall survival in gastric cancer patients (16).

In breast cancer, PHLDA1 was identified among the gene set of oncogenic ras-pathway and interferon-response 
signatures in selected small populations of cluster of differentiation (CD) $24^{-/ \mathrm{low}} / \mathrm{CD} 44^{+}$stem cells from several breast cancer cell lines (46). More recently, high PHLDA1 expression was shown to contribute to the survival and growth of breast cancer stem cells (41). Kastrati et al (41) also found upregulation of PHLDA1 mRNA and protein expression in a panel of estrogen receptor-positive breast cancer cells that were growing in mammosphere cultures compared to cells growing in adherent monolayer cultures. The study also observed that PHLDAl knockdown affects mammosphere formation and growth, leading to a reduction in the number and size of the mammospheres.

\section{Conclusions}

Over the last few years, PHLDA1 has attracted increasing attention. However, the data reviewed herein show that the precise molecular and cellular functions of PHLDA1 remain to be fully elucidated. Although several lines of evidence have implicated PHLDA1 as a potential transcriptional activator that acts as a proapoptotic and antiproliferative factor, the exact mechanisms by which PHLDA1 mediates cell survival are still under investigation. PHLDA1 expression can be modulated by various stimuli, resulting in pleiotropic effects, with upregulation and downregulation of different biological processes, such as proliferation, apoptosis, migration and invasion, which are considered key hallmarks of carcinogenesis. The pleiotropic effects of PHLDA1 indicate tissue-specific activities that depend on the cellular context. PHLDA1 is expressed in different tissues with variable subcellular localization and function, and its expression pattern is altered in different types of tumors. Data gathered thus far indicate that PHLDA1 has an important role in the tumorigenic process; however, new results from clinical and experimental studies are necessary to improve the definition of its role as a tumor suppressor gene or oncogene and its contribution to tumor progression. Further, well-designed clinical and experimental studies, in vitro and in vivo, that use informative and testable animal models are required to improve the understanding of the PHLDA1 mechanisms of action in normal and cancer cells. In addition, the identification of PHLDA1 as a potential stem cell marker and evidence of its possible roles in the EMT indicate that there are new perspectives and research fields to be explored to evaluate the actual contributions of PHLDA1 to the tumorigenic process and its role not only as prognostic factor, but also as a potential therapeutic target.

\section{References}

1. Park CG, Lee SY, Kandala G, Lee SY and Choi Y: A novel gene product that couples TCR signaling to Fas(CD95) expression in activation-induced cell death. Immunity 4: 583-591, 1996.

2. Kuske MD and Johnson JP: Assignment of the human PHLDA1 gene to chromosome $12 \mathrm{q} 15$ by radiation hybrid mapping. Cytogenet Cell Genet 89: 1, 2000.

3. Frank D, Mendelsohn CL, Ciccone E, Svensson K, Ohlsson R and Tycko B: A novel pleckstrin homology-related gene family defined by Ipl/Tssc3, TDAG51, and Tih1: Tissue-specific expression, chromosomal location, and parental imprinting. Mamm Genome 10: 1150-1159, 1999.

4. Gomes I, Xiong W, Miki T and Rosner MR: A proline- and glutamine-rich protein promotes apoptosis in neuronal cells. J Neurochem 73: 612-622, 1999.
5. Lemmon MA, Ferguson KM and Abrams CS: Pleckstrin homology domains and the cytoskeleton. FEBS Lett 513: 71-76, 2002.

6. Xu Q, Bateman A, Finn RD, Abdubek P, Astakhova T, Axelrod HL, Bakolitsa C, Carlton D, Chen C, Chiu HJ, et al: Bacterial pleckstrin homology domains: A prokaryotic origin for the PH domain. J Mol Biol 396: 31-46, 2010.

7. Lemmon MA and Ferguson KM: Signal-dependent membrane targeting by pleckstrin homology $(\mathrm{PH})$ domains. Biochem J 350: $1-18,2000$.

8. Scheffzek K and Welti S: Pleckstrin homology (PH) like domains - versatile modules in protein-protein interaction platforms. FEBS Lett 586: 2662-2673, 2012.

9. Williamson MP: The structure and function of proline-rich regions in proteins. Biochem J 297: 249-260, 1994.

10. Butland SL, Devon RS, Huang Y, Mead CL, Meynert AM, Neal SJ, Lee SS, Wilkinson A, Yang GS, Yuen MM, et al: CAG-encoded polyglutamine length polymorphism in the human genome. BMC Genomics 8: 126, 2007.

11. Liu CR and Cheng TH: Allele-selective suppression of mutant genes in polyglutamine diseases. J Neurogenet 29: 41-49, 2015.

12. Hossain GS, van Thienen JV, Werstuck GH, Zhou J, Sood SK, Dickhout JG, de Koning AB, Tang D, Wu D, Falk E, et al: TDAG51 is induced by homocysteine, promotes detachment-mediated programmed cell death, and contributes to the cevelopment of atherosclerosis in hyperhomocysteinemia. J Biol Chem 278: 30317-30327, 2003.

13. Hinz T, Flindt S, Marx A, Janssen O and Kabelitz D: Inhibition of protein synthesis by the $\mathrm{T}$ cell receptor-inducible human TDAG51 gene product. Cell Signal 13: 345-352, 2001.

14. Neef R, Kuske MA, Pröls E and Johnson JP: Identification of the human PHLDA1/TDAG51 gene: Down-regulation in metastatic melanoma contributes to apoptosis resistance and growth deregulation. Cancer Res 62: 5920-5929, 2002.

15. Nagai MA, Fregnani JH, Netto MM, Brentani MM and Soares FA: Down-regulation of PHLDA1 gene expression is associated with breast cancer progression. Breast Cancer Res Treat 106: 49-56, 2007.

16. Zhao P, Lu Y and Liu L: Correlation of decreased expression of PHLDA1 protein with malignant phenotype of gastric adenocarcinoma. Int J Clin Exp Pathol 8: 5230-5235, 2015.

17. Sakthianandeswaren A, Christie M, D'Andreti C, Tsui C, Jorissen RN, Li S, Fleming NI, Gibbs P, Lipton L, Malaterre J, et al: PHLDA1 expression marks the putative epithelial stem cells and contributes to intestinal tumorigenesis. Cancer Res 71: 3709-3719, 2011.

18. Mohammad RM, Muqbil I, Lowe L, Yedjou C, Hsu HY, Lin LT, Siegelin MD, Fimognari C, Kumar NB, Dou QP, et al: Broad targeting of resistance to apoptosis in cancer. Semin Cancer Biol 35: S78-S103, 2015.

19. Rho J, Gong S, Kim N and Choi Y: TDAG51 is not essential for Fas/CD95 regulation and apoptosis in vivo. Mol Cell Biol 21: 8365-8370, 2001 .

20. Oberg HH, Sipos B, Kalthoff H, Janssen O and Kabelitz D: Regulation of T-cell death-associated gene 51 (TDAG51) expression in human T-cells. Cell Death Differ 11: 674-684, 2004.

21. Wang R, Zhang L, Yin D, Mufson RA and Shi Y: Protein kinase $C$ regulates Fas (CD95/APO-1) expression. J Immunol 161: 2201-2207, 1998.

22. Joo JH, Liao G, Collins JB, Grissom SF and Jetten AM: Farnesol-induced apoptosis in human lung carcinoma cells is coupled to the endoplasmic reticulum stress response. Cancer Res 67: 7929-7936, 2007.

23. Carlisle RE, Heffernan A, Brimble E, Liu L, Jerome D, Collins CA, Mohammed-Ali Z, Margetts PJ, Austin RC and Dickhout JG: TDAG51 mediates epithelial-to-mesenchymal transition in human proximal tubular epithelium. Am J Physiol Renal Physiol 303: F467-F481, 2012.

24. Shang Y, Cai X and Fan D: Roles of epithelial-mesenchymal transition in cancer drug resistance. Curr Cancer Drug Targets 13: 915-929, 2013.

25. Pasquier J, Abu-Kaoud N, Al Thani H and Rafii A: Epithelial to Mesenchymal Transition in a Clinical Perspective. J Oncol 2015: 792182, 2015.

26. Moad AI, Muhammad TS, Oon CE and Tan ML: Rapamycin induces apoptosis when autophagy is inhibited in T-47D mammary cells and both processes are regulated by Phlda1. Cell Biochem Biophys 66: 567-587, 2013. 
27. Hayashida N, Inouye S, Fujimoto M, Tanaka Y, Izu H, Takaki E, Ichikawa H, Rho J and Nakai A: A novel HSF1-mediated death pathway that is suppressed by heat shock proteins. EMBO J 25: 4773-4783, 2006.

28. Liu F, Xu ZL, Qian XJ, Qiu WY and Huang H: Expression of Hsf1, Hsf2, and Phlda1 in cells undergoing cryptorchid-induced apoptosis in rat testes. Mol Reprod Dev 78: 283-291, 2011.

29. Toyoshima Y, Karas M, Yakar S, Dupont J, Lee Helman and LeRoith D: TDAG51 mediates the effects of insulin-like growth factor I (IGF-I) on cell survival. J Biol Chem 279: 25898-25904, 2004.

30. Wu S, Walenkamp MJ, Lankester A, Bidlingmaier M, Wit JM and De Luca F: Growth hormone and insulin-like growth factor I insensitivity of fibroblasts isolated from a patient with an IкB $\alpha$ mutation. J Clin Endocrinol Metab 95: 1220-1228, 2010.

31. Boro A, Prêtre K, Rechfeld F, Thalhammer V, Oesch S, Wachtel M, Schäfer BW and Niggli FK: Small-molecule screen identifies modulators of EWS/FLI1 target gene expression and cell survival in Ewing's sarcoma. Int J Cancer 131: 2153-2164, 2012.

32. Sohn EJ, Li H, Reidy K, Beers LF, Christensen BL and Lee SB: EWS/FLI1 oncogene activates caspase 3 transcription and triggers apoptosis in vivo. Cancer Res 70: 1154-1163, 2010.

33. Murata T, Sato T, Kamoda T, Moriyama H, Kumazawa Y and Hanada N: Differential susceptibility to hydrogen sulfide-induced apoptosis between PHLDA1-overexpressing oral cancer cell lines and oral keratinocytes: Role of PHLDA1 as an apoptosis suppressor. Exp Cell Res 320: 247-257, 2014.

34. Hanahan D and Weinberg RA: Hallmarks of cancer: The next generation. Cell 144: 646-674, 2011

35. Johnson EO, Chang KH, de Pablo Y, Ghosh S, Mehta R, Badve S and Shah K: PHLDA1 is a crucial negative regulator and effector of Aurora A kinase in breast cancer. J Cell Sci 124: 2711-2722, 2011.

36. Li G, Wang X, Hibshoosh H, Jin C and Halmos B: Modulation of ErbB2 blockade in ErbB2-positive cancers: The role of ErbB2 Mutations and PHLDA1. PLoS One 9: e106349, 2014.

37. Coutinho-Camillo CM, Lourenco SV, Nonogaki S, Vartanian JG, Nagai MA, Kowalski LP and Soares FA: Expression of PAR-4 and PHLDA1 is prognostic for overall and disease-free survival in oral squamous cell carcinomas. Virchows Arch 463: 31-39, 2013.
38. Chiu ST, Hsieh FJ, Chen SW, Chen CL, Shu HF and Li H Clinicopathologic correlation of up-regulated genes identified using cDNA microarray and real-time reverse transcription-PCR in human colorectal cancer. Cancer Epidemiol Biomarkers Prev 14: 437-443, 2005.

39. Ren L, Mendoza A, Zhu J, Briggs JW, Halsey C, Hong ES, Burkett SS, Morrow J, Lizardo MM, Osborne T, et al: Characterization of the metastatic phenotype of a panel of established osteosarcoma cells. Oncotarget 6: 29469-29481, 2015.

40. Marchiori AC, Casolari DA and Nagai MA: Transcriptional up-regulation of PHLDA1 by 17beta-estradiol in MCF-7 breast cancer cells. Braz J Med Biol Res 41: 579-582, 2008.

41. Kastrati I, Canestrari E and Frasor J: PHLDA1 expression is controlled by an estrogen receptor-NFkB-miR-181 regulatory loop and is essential for formation of ER+ mammospheres. Oncogene 34: 2309-2316, 2015.

42. Oberst MD, Beberman SJ, Zhao L, Yin JJ, Ward Y and Kelly K: TDAG51 is an ERK signaling target that opposes ERK-mediated HME16C mammary epithelial cell transformation. BMC Cancer 8: 189-204, 2008.

43. Ohyama M, Terunuma A, Tock CL, Radonovich MF, Pise-Masison CA, Hopping SB, Brady JN, Udey MC and Vogel JC: Characterization and isolation of stem cell-enriched human hair follicle bulge cells. J Clin Invest 116: 249-260, 2006.

44. Sellheyer K and Krahl D: PHLDA1 (TDAG51) is a follicular stem cell marker and differentiates between morphoeic basal cell carcinoma and desmoplastic trichoepithelioma. Br J Dermatol 164: 141-147, 2011.

45. Guezguez A, Paré F, Benoit YD, Basora N and Beaulieu JF: Modulation of stemness in a human normal intestinal epithelial crypt cell line by activation of the WNT signaling pathway. Exp Cell Res 322: 355-364, 2014.

46. Murohashi M, Hinohara K, Kuroda M, Isagawa T, Tsuji S, Kobayashi S, Umezawa K, Tojo A, Aburatani H and Gotoh N: Gene set enrichment analysis provides insight into novel signalling pathways in breast cancer stem cells. Br J Cancer 102: 206-212,2010. 